\title{
Photonic crystal-based compact high-power vacuum electronic devices
}

\author{
V. G. Baryshevsky and A. A. Gurinovich \\ Research Institute for Nuclear Problems, 220030 Minsk, Belarus
}

(Received 10 December 2018; published 30 April 2019)

\begin{abstract}
This paper considers how the finite dimensions of a photonic crystal placed inside a resonator or waveguide affect the law of electron beam instability. The dispersion equations describing e-beam instability in the finite photonic crystal placed inside the resonator or waveguide (a bounded photonic crystal) are obtained. Application of photonic crystals for development of $\mathrm{THz}$ sources at electron beam current densities available at modern accelerators is discussed.
\end{abstract}

DOI: 10.1103/PhysRevAccelBeams.22.044702

\section{INTRODUCTION}

$\mathrm{THz}$ sources are in demand for a variety of applications: information and communications technology, biology and medicine, nondestructive investigations and homeland security, food and agricultural products quality control, global environmental monitoring, space research and ultrafast computing. High-power tunable $\mathrm{THz}$ sources are very important devices to bring promising prospects to wide use; high efficiency and compactness are also highly desired (see $[1,2]$ and references therein).

Sources of THz radiation are studied by numerous authors through expansion to THz range of approaches and principles, which generally serve for microwave sources; namely, traveling wave tubes, backward wave oscillators, generators utilizing diffraction or Smith-Purcell radiation, etc. [3-6]. A slow-wave structure (SWS) is generally used in such devices to make the electromagnetic wave phase velocity less than the speed of light so that beam electrons can interact with the wave and convert their energy to radiation. A diffraction grating, a helical line, a corrugated waveguide, a photonic crystal, a multipin structure or a spatially periodic structure of any type, they all could work as SWSs and enable frequency tuning by a change of their geometry [7-11].

The general feature for all the above listed radiation sources is the instability of an electron beam in a spatially periodic media, which results in beam self-modulation and radiation of electromagnetic waves. The increment $\delta_{0} \sim$ $\operatorname{Im} k_{z}$ ( $k_{z}$ is the longitudinal wave number), which describes the electron beam instability responsible for radiation process in such devices, conventionally is determined by the unperturbed density $\rho_{b 0}$ of electrons in the beam as

Published by the American Physical Society under the terms of the Creative Commons Attribution 4.0 International license. Further distribution of this work must maintain attribution to the author(s) and the published article's title, journal citation, and DOI. follows: $\delta_{0} \sim \sqrt[3]{\rho_{b 0}}[12-14]$. The threshold current density $j_{\text {thr }}$, which is required for coherent electromagnetic oscillations to grow, in this case depends on the beam-wave interaction length $L$ as $j_{\text {thr }} \sim L^{-3}$.

The shift to $\mathrm{THz}$ range for conventional microwave devices faces several difficulties limiting the output power due to a drastic decrease in dimensions of the interaction area. The SWS period tends to submillimeter range and requirements to electron beam quality and guiding precision become more strict. The amplitude of the harmonic, which is in synchronism with the beam, decreases with the distance from the SWS surface on the scale

$$
\Delta=\frac{\lambda \beta \gamma}{2 \pi}
$$

where $\lambda$ is the wavelength, $\beta=\frac{v}{c}, \gamma$ is the Lorentz factor, and $v$ and $c$ are the speed of electron beam and light, respectively. Thus, only electrons moving close to the structure can efficiently interact with the wave; for example, $\Delta \approx 0.1 \mathrm{~mm}$ for $100 \mathrm{keV}$ electron beam and $\lambda=1 \mathrm{~mm}$. Small dimensions constrain applicable electron beam currents and, therefore, available output power. Therefore, approaches enabling increase of efficiency and transverse dimensions of interaction area (electronbeam cross section) are of high priority for $\mathrm{THz}$ source development. Such approaches were for the first time ever proposed for $\mathrm{x}$-ray range by the concept of volume free electron laser (VFEL) [9,15-17], which enables increase of both the efficiency and the transverse coherence area, and simultaneous reduction of threshold current density and operation length. Successive expansion of the VFEL concept to microwave $[5-8,18]$, terahertz $[19,20]$ and visible light [21] ranges includes both theoretical and experimental studies; diversely designed SWS are used; namely, diffraction gratings, photonic crystals, etc.

Another law of electron beam instability was discovered in $[9,15-17]$. It was found there that for an electron beam 
moving in a crystal for the case, when Bragg diffraction could occur, the electron beam instability increment $\delta_{0}$ could turn out to be determined by $\delta_{0} \sim \sqrt[4]{\rho_{b 0}}$ rather than conventional $\delta_{0} \sim \sqrt[3]{\rho_{b 0}}$ [12-14]. The law of electron beam instability inherent for the volume free electron laser $[9,15-17]$ is revealed when distributed feedback is formed by Bragg diffraction of the beam-induced electromagnetic wave by a spatially periodic SWS. This law gives for the threshold current density the very different dependence on interaction length $L$ as follows: $j_{\text {thr }} \sim L^{-3-2 s}$, where $s$ is the number of additional waves arisen due to diffraction (when one additional wave arises then $j_{\mathrm{thr}} \sim L^{-5}$ ).

For a certain slow-wave structure this law reveals only for the narrow range of parameters (electron beam energy and radiation frequency), for which the group velocity of the excited wave is close to zero, i.e., synchronism of beam and wave is achieved in the vicinity of $\pi$-point on the SWS dispersion curve $k(\omega)=0$ ( $k$ is the wave number, $\omega$ is the radiation frequency). For example, when in addition to the electromagnetic wave, which is excited by electron beam via its interaction with SWS, one more wave, which propagates in the direction determined by the Bragg's law, is present, one could expect that the threshold current density $j_{\text {thr }}$ is defined by law $j_{\text {thr }} \sim L^{-5}$ rather than $j_{\text {thr }} \sim L^{-3}$ for proper combination of electron beam energy and radiation frequency. In particular, the law $j_{\text {thr }} \sim L^{-5}$ can be observed in the conventional backward wave oscillator with corrugated waveguide in the case when the excited wave and the diffracted one propagate in opposite directions along the system axis.

First lasing of VFEL, which uses the above described instability law, was presented in [7]. Frequency tuning in this generator is analyzed in [8]. The theoretical study of the instability of electron beams moving in natural and artificial (photonic) crystals was carried out for the ideal case of an infinite medium (see the review [9] and $[5,7,15,18,22])$. It is known that the discrete structure of the modes in waveguides and resonators is crucial for effective generation in the microwave range [23-25]. This paper considers how the finite dimensions of a photonic crystal placed inside a resonator or waveguide affect the law of electron beam instability.

The dispersion equation describing e-beam instability in the finite photonic crystal placed inside the resonator or waveguide (a bounded photonic crystal) is obtained. The instability law is shown to be valid and caused by mixing of the electromagnetic field modes in the finite volume due to the periodic disturbance produced by the photonic crystal.

\section{EQUATIONS DESCRIBING MOTION OF A RELATIVISTIC ELECTRON BEAM IN A BOUNDED PHOTONIC CRYSTAL}

To describe generation of induced radiation (i.e., electron beam instability) in either a photonic or a natural crystal one should start from Maxwell equations: $\operatorname{rot} \vec{H}(\vec{r}, t)=\frac{1}{c} \frac{\partial \vec{D}(\vec{r}, t)}{\partial t}+\frac{4 \pi}{c} \vec{j}(\vec{r}, t)$,

$\operatorname{rot} \vec{E}(\vec{r}, t)=-\frac{1}{c} \frac{\partial \vec{H}(\vec{r}, t)}{\partial t}$,

$\operatorname{div} \vec{D}(\vec{r}, t)=4 \pi \rho(\vec{r}, t), \quad \frac{\partial \rho(\vec{r}, t)}{\partial t}+\operatorname{div} \vec{j}(\vec{r}, t)=0$,

where $\vec{E}(\vec{r}, t)$ and $\vec{H}(\vec{r}, t)$ are the strength of the electric and the magnetic field, respectively; $\vec{j}(\vec{r}, t)$ and $\rho(\vec{r}, t)$ are the current and charge densities; $D_{i}(\vec{r}, t)=\int \varepsilon_{i l}\left(\vec{r}, t, t^{\prime}\right)$ $E_{l}\left(\vec{r}, t^{\prime}\right) d t^{\prime}$, where indices $i, l=1,2,3$ correspond to $x$, $y, z$; in the case of properties of the photonic crystal not depending on time, its dielectric permittivity tensor reads as $\varepsilon_{i l}\left(\vec{r}, t-t^{\prime}\right)=\frac{1}{2 \pi} \int_{-\infty}^{+\infty} \varepsilon_{i l}(\vec{r}, \omega) e^{-i \omega\left(t-t^{\prime}\right)} d \omega$.

The current and charge densities are defined as

$$
\begin{aligned}
& \vec{j}(\vec{r}, t)=e \sum_{\alpha} \vec{v}_{\alpha} \delta\left(\vec{r}-\vec{r}_{\alpha}(t)\right), \\
& \rho(\vec{r}, t)=e \sum_{\alpha} \delta\left(\vec{r}-\vec{r}_{\alpha}(t)\right)=e \rho_{b}(\vec{r}, t),
\end{aligned}
$$

where $e$ is the electron charge, and $\rho_{b}(\vec{r}, t)$ is the beam density (the number of electrons per $1 \mathrm{~cm}^{3}$ ). The velocity $\vec{v}_{\alpha}=\vec{v}_{\alpha}(t)$ of the electron with number $\alpha$ can be obtained by the relativistic equation of charge motion in an electromagnetic field (see Sec. 17 in [26]):

$$
\frac{d \vec{p}_{\alpha}}{d t}=m \frac{d}{d t}\left(\gamma_{\alpha} \vec{v}_{\alpha}\right)=e\left\{\vec{E}\left(\vec{r}_{\alpha}, t\right)+\frac{1}{c}\left[\vec{v}_{\alpha} \times \vec{H}\left(\vec{r}_{\alpha}, t\right)\right]\right\},
$$

where $\vec{p}_{\alpha}=\vec{p}_{\alpha}(t)$ is the particle momentum, $\gamma_{\alpha}=\left(1-\frac{v_{\alpha}^{2}}{c^{2}}\right)^{-\frac{1}{2}}$ is the Lorentz factor, and $\vec{E}\left(\vec{r}_{\alpha}, t\right)$ and $\vec{H}\left(\vec{r}_{\alpha}, t\right)$ are the electric and magnetic field strength at point $\vec{r}_{\alpha}=\vec{r}_{\alpha}(t)$, where the electron with number $\alpha$ is located.

Following the exercise concluding Sec. 17 in [26], one can transform (4) to the equation for $\vec{v}_{\alpha}$ as follows:

$$
\begin{aligned}
\frac{d \vec{v}_{\alpha}}{d t}= & \frac{e}{m \gamma_{\alpha}}\left\{\vec{E}\left(\vec{r}_{\alpha}, t\right)+\frac{1}{c}\left[\vec{v}_{\alpha} \times \vec{H}\left(\vec{r}_{\alpha}, t\right)\right]\right. \\
& \left.-\frac{\vec{v}_{\alpha}}{c^{2}}\left[\vec{v}_{\alpha} \vec{E}\left(\vec{r}_{\alpha}, t\right)\right]\right\} .
\end{aligned}
$$

From Eq. (2) one can obtain

$$
-\Delta \vec{E}(\vec{r}, t)+\vec{\nabla}[\vec{\nabla} \vec{E}(\vec{r}, t)]+\frac{1}{c^{2}} \frac{\partial^{2} \vec{D}(\vec{r}, t)}{\partial t^{2}}=-\frac{4 \pi}{c^{2}} \frac{\partial \vec{j}(\vec{r}, t)}{\partial t} .
$$

The description of radiation generation and instability of electron beam moving in the photonic crystal becomes much simpler in a practically important case, when all the 
elements $\chi_{i k}$ of tensor $\hat{\chi}$ are much smaller as compared to the unity $\left|\chi_{i k}\right| \ll 1$. In this case Eq. (6) can be rewritten as follows:

$$
\begin{gathered}
\Delta \vec{E}(\vec{r}, t)-\frac{1}{c^{2}} \frac{\partial^{2}}{\partial t^{2}} \int \hat{\varepsilon}\left(\vec{r}, t-t^{\prime}\right) \vec{E}\left(\vec{r}, t^{\prime}\right) d t^{\prime} \\
=4 \pi\left(\frac{1}{c^{2}} \frac{\partial \vec{j}(\vec{r}, t)}{\partial t}+\vec{\nabla} \rho(\vec{r}, t)\right) .
\end{gathered}
$$

For detailed justification of its validity see the Appendix.

In the general case the dielectric permittivity tensor can be presented in the form $\hat{\varepsilon}(\vec{r})=1+\hat{\chi}(\vec{r})$, where $\hat{\chi}(\vec{r})$ is the susceptibility of the photonic crystal

$$
\hat{\chi}(\vec{r})=\sum_{i} \hat{\chi}_{\text {cell }}\left(\vec{r}-\vec{r}_{i}\right),
$$

where $\hat{\chi}_{\text {cell }}\left(\vec{r}-\vec{r}_{i}\right)$ is the susceptibility of the crystal unit cell. The susceptibility of an infinite perfect crystal $\hat{\chi}(\vec{r})$ can be expanded into a Fourier series as follows: $\hat{\chi}(\vec{r})=\sum_{\vec{\tau}} \hat{\chi}_{\vec{\tau}} e^{i \vec{\tau} \vec{r}}$, where $\vec{\tau}$ is the reciprocal lattice vector of the crystal.
Let us consider in detail a practically important case of a bounded photonic crystal; to be more specific, let us study a photonic crystal placed inside a waveguide of rectangular cross section with smooth walls. The walls of the waveguide are assumed to be perfectly conductive. The eigenfunctions and eigenvalues of a rectangular waveguide are well studied $[27,28]$. Suppose the $z$ axis to be directed along the waveguide axis, $a$ and $b$ are the waveguide dimensions along $x$ and $y$ axes, respectively. Let us make a Fourier transform of (6) over time and longitudinal coordinate $z$. In this case $D_{i}(\vec{r}, \omega)=$ $\varepsilon_{i l}(\vec{r}, \omega) E_{l}(\vec{r}, \omega)$. Expanding the obtained equation for the field $\vec{E}\left(\vec{r}_{\perp}, k_{z}, \omega\right)$ over a full set of vector eigenfunctions $\vec{Y}_{m n}^{\lambda}\left(\vec{r}_{\perp}, k_{z}\right)$ of a rectangular waveguide (where $m, n=1,2,3 \ldots$ and $\lambda$ describes the type of the wave [29]), one can obtain for field $\vec{E}$ the equality

$$
\vec{E}\left(\vec{r}_{\perp}, k_{z}, \omega\right)=\sum_{m n \lambda} C_{m n}^{\lambda}\left(k_{z}, \omega\right) \vec{Y}_{m n}^{\lambda}\left(\vec{r}_{\perp}, k_{z}\right) .
$$

As a result, the following system of equations can be written:

$$
\begin{aligned}
& {\left[\left(k_{z}^{2}+\varkappa_{m n \lambda}^{2}\right)-\frac{\omega^{2}}{c^{2}}\right] C_{m n}^{\lambda}\left(k_{z}, \omega\right)-\frac{\omega^{2}}{c^{2}} \frac{1}{2 \pi} \sum_{m^{\prime} n^{\prime} \lambda^{\prime}} \int \vec{Y}_{m n}^{\lambda^{*}}\left(\vec{r}_{\perp}, k_{z}\right) \hat{\chi}(\vec{r}) \vec{Y}_{m^{\prime} n^{\prime}}^{\lambda^{\prime}}\left(\vec{r}_{\perp}, k_{z}^{\prime}\right) e^{-i\left(k_{z}-k_{z}^{\prime}\right) z} d^{2} r_{\perp} C_{m^{\prime} n^{\prime}}^{\lambda^{\prime}}\left(k_{z}^{\prime}, \omega\right) d k_{z}^{\prime} d z} \\
& \quad=\frac{4 \pi i \omega}{c^{2}} \int \vec{Y}_{m n}^{\lambda^{*}}\left(\vec{r}_{\perp}, k_{z}\right)\left\{\vec{j}\left(\vec{r}_{\perp}, z, \omega\right)+\frac{c^{2}}{\omega^{2}} \vec{\nabla}\left[\vec{\nabla} \vec{j}\left(\vec{r}_{\perp}, z, \omega\right)\right]\right\} e^{-i k_{z} z} d^{2} r_{\perp} d z,
\end{aligned}
$$

where $\varkappa_{m n \lambda}^{2}=k_{x m \lambda}^{2}+k_{y n \lambda}^{2}$.

The beam current and density, which appear on the righthand side of (10), are the complicated functions of $\vec{E}(\vec{r}, \omega)$. To study the system instability, it is sufficient to consider it in the approximation linear over perturbation, i.e., one can expand the expressions for $\vec{j}(\vec{r}, \omega)$ and $\rho(\vec{r}, \omega)$ over $\vec{E}(\vec{r}, \omega)$ amplitude and confine oneself with the linear approximation [12-14,30,31].

As a result, a closed system of equations comes out. For further consideration, one should find the expressions for corrections to beam current density $\delta \vec{j}(\vec{r}, \omega)$ and beam charge density $\delta \rho(\vec{r}, \omega)$, which arise due to beam perturbation by the field. Considering the Fourier transforms of current and charge densities $\vec{j}(\vec{k}, \omega)$ and $\rho(\vec{k}, \omega)$, one can obtain from (3) that

$$
\begin{aligned}
\delta \vec{j}(\vec{k}, \omega)= & e \sum_{\alpha=1}^{N} e^{-i \vec{k} \vec{r}_{\alpha_{0}}}\left\{\delta \vec{v}_{\alpha}\left(\omega-\vec{k} \vec{u}_{\alpha}\right)\right. \\
& \left.+\vec{u}_{\alpha} \frac{\vec{k} \delta \vec{v}_{\alpha}\left(\omega-\vec{k} \vec{u}_{\alpha}\right)}{\omega-\vec{k} \vec{u}_{\alpha}}\right\}
\end{aligned}
$$

where $\vec{r}_{\alpha_{0}}$ is the initial coordinate of the electron, and $\vec{u}_{\alpha}$ is the unperturbed velocity of the electron.

For simplicity, let us consider a cold beam, for which $\vec{u}_{\alpha} \approx \vec{u}$, where $\vec{u}$ is the mean velocity of the beam. The general case of a hot beam can be obtained by averaging $\delta \vec{j}(\vec{k}, \omega)$ over distribution of particle the velocities $\vec{u}_{\alpha}$ in the beam.

According to (5), velocity $\delta \vec{v}_{\alpha}$ is determined by field $\vec{E}\left(\vec{r}_{\alpha}, \omega\right)$. The Fourier transform of $\vec{E}\left(\vec{r}_{\alpha}, \omega\right)$ has a form

$$
\vec{E}\left(\vec{r}_{\alpha}, \omega\right)=\frac{1}{(2 \pi)^{3}} \int \vec{E}\left(\vec{k}^{\prime}, \omega\right) e^{i \vec{k}^{\prime} \vec{r}_{\alpha}} d^{3} k^{\prime}
$$

As a result, $\delta \vec{j}(\vec{k}, \omega)$ includes the following sum over the beam particles $\sum_{\alpha} e^{-i\left(\vec{k}-\vec{k}^{\prime}\right) \vec{r}_{\alpha}}$. Suppose that the electrons in an unperturbed beam are uniformly distributed over the area occupied by the beam. Therefore

$$
\sum_{\alpha} e^{-i\left(\vec{k}-\vec{k}^{\prime}\right) \vec{r}_{\alpha}}=(2 \pi)^{3} \rho_{b 0} \delta\left(\vec{k}-\vec{k}^{\prime}\right) .
$$

As a result, the following expression for $\delta \vec{j}(\vec{k}, \omega)$ can be obtained [32,33]: 


$$
\delta \vec{j}(\vec{k}, \omega)=i \frac{\vec{u} e^{2} \rho_{b 0}\left(k^{2}-\frac{\omega^{2}}{c^{2}}\right)}{(\omega-\vec{k} \vec{u})^{2} m \gamma \omega} \vec{u} \vec{E}(\vec{k}, \omega) .
$$

One can obtain the expression for $\rho_{b}(\vec{k}, \omega)$ using the continuity equation. Expression (12), the inverse Fourier transform of $\vec{E}(\vec{k}, \omega)$, and the expansion (9) enable writing the system of Eq. (10) as follows:

$$
\begin{aligned}
& {\left[\left(k_{z}^{2}+\varkappa_{m n \lambda}^{2}\right)-\frac{\omega^{2}}{c^{2}}\right] C_{m n}^{\lambda}\left(k_{z}, \omega\right)-\frac{\omega^{2}}{c^{2}} \frac{1}{2 \pi} \sum_{m^{\prime} n^{\prime} \lambda^{\prime}} \int \vec{Y}_{m n}^{\lambda^{*}}\left(\vec{r}_{\perp}, k_{z}\right) \hat{\chi}(\vec{r}) \vec{Y}_{m^{\prime} n^{\prime}}^{\lambda^{\prime}}\left(\vec{r}_{\perp}, k_{z}^{\prime}\right) e^{-i\left(k_{z}-k_{z}^{\prime}\right) z} d^{2} r_{\perp} C_{m^{\prime} n^{\prime}}^{\lambda^{\prime}}\left(k_{z}^{\prime}, \omega\right) d k_{z}^{\prime} d z} \\
& =-\frac{\omega_{L}^{2}\left(k_{m n}^{2} c^{2}-\omega^{2}\right)}{\gamma c^{4}\left(\omega-\vec{k}_{m n} \vec{u}\right)^{2}}\left\{\frac{1}{2 \pi}\left|\int \vec{u} \vec{Y}_{m n}^{\lambda}\left(\vec{k}_{\perp}, k_{z}\right) d^{2} k_{\perp}\right|^{2}\right\} C_{m n}^{\lambda}\left(k_{z}, \omega\right),
\end{aligned}
$$

where $\vec{Y}_{m n}^{\lambda}\left(\vec{k}_{\perp}, k_{z}\right)=\int e^{-i \vec{k}_{\perp} \vec{r}_{\perp}} \vec{Y}_{m n}^{\lambda}\left(\vec{r}_{\perp}, k_{z}\right) d^{2} r_{\perp}$.

The system of Eq. (13) in the approximation linear over perturbation describes the electromagnetic field modes, which are induced by an electron beam in the finite volume of a rectangular waveguide due to the periodic disturbance produced by a photonic crystal.

\section{RADIATIVE INSTABILITY OF A RELATIVISTIC ELECTRON BEAM MOVING IN A BOUNDED PHOTONIC CRYSTAL}

The above obtained system of Eq. (13) enables to derive the dispersion equation for a bounded photonic crystal and to analyze conditions, when electron beam instability presents. Let us consider the sums in the left-hand side of Eq. (13):

$$
\begin{aligned}
& \sum_{m^{\prime} n^{\prime} \lambda^{\prime}} \int \vec{Y}_{m n}^{\lambda^{*}}\left(\vec{r}_{\perp}, k_{z}\right) \hat{\chi}(\vec{r}) \vec{Y}_{m^{\prime} n^{\prime}}^{\lambda^{\prime}}\left(\vec{r}_{\perp}, k_{z}^{\prime}\right) e^{-i\left(k_{z}-k_{z}^{\prime}\right) z} d^{2} r_{\perp} C_{m^{\prime} n^{\prime}}^{\lambda^{\prime}}\left(k_{z}^{\prime}, \omega\right) d k_{z}^{\prime} d z \\
& \quad=\sum_{m^{\prime} n^{\prime} \lambda^{\prime}} \int C_{m^{\prime} n^{\prime}}^{\lambda^{\prime}}\left(k_{z}^{\prime}, \omega\right) \int \vec{Y}_{m n}^{\lambda^{*}}\left(\vec{r}_{\perp}, k_{z}\right) \hat{\chi}(\vec{r}) \vec{Y}_{m^{\prime} n^{\prime}}^{\lambda^{\prime}}\left(\vec{r}_{\perp}, k_{z}^{\prime}\right) e^{-i\left(k_{z}-k_{z}^{\prime}\right) z} d^{2} r_{\perp} d z d k_{z}^{\prime}
\end{aligned}
$$

and analyze integrals

$$
\int \vec{Y}_{m n}^{\lambda^{*}}\left(\vec{r}_{\perp}, k_{z}\right) \hat{\chi}(\vec{r}) \vec{Y}_{m^{\prime} n^{\prime}}^{\lambda^{\prime}}\left(\vec{r}_{\perp}, k_{z}^{\prime}\right) e^{-i\left(k_{z}-k_{z}^{\prime}\right) z} d^{2} r_{\perp} d z
$$

to evaluate what terms mostly contribute to the considered sums. Using (8) and representing eigenfunctions $\vec{Y}_{m n}^{\lambda}\left(\vec{r}_{\perp}, k_{z}\right)$ of a rectangular waveguide [27-29] by combinations of sines and cosines of the form $\sin \frac{\pi m}{a} x, \cos \frac{\pi m}{a} x$, $\sin \frac{\pi n}{b} y, \cos \frac{\pi n}{b} y$ (i.e., in fact, the combinations $e^{i \frac{i m}{a} x}, e^{i \frac{\pi n}{b} y}$ ) integrals (15) can be transformed to the expressions of the form as follows:

$$
\begin{aligned}
& I_{x}=\int e^{-i \frac{\pi m}{a} x} \sum_{i} \hat{\chi}_{\text {cell }}\left(x-x_{i}, y-y_{i}, z-z_{i}\right) e^{i \frac{\pi m^{\prime}}{a} x} d x, \\
& I_{y}=\int e^{-i \frac{\pi m}{b} y} \sum_{i} \hat{\chi}_{\text {cell }}\left(x-x_{i}, y-y_{i}, z-z_{i}\right) e^{i \frac{\pi{ }^{\prime}}{b} y} d y .
\end{aligned}
$$

Substitution of variables $x-x_{i}=\eta_{1}$ in (16) and $y-y_{i}=$ $\eta_{2}$ in (17) produces the sums of the form

$$
S_{x}=\sum_{f_{1}=1}^{N_{x}} e^{-i \frac{\pi}{a}\left(m-m^{\prime}\right) d_{x} f_{1}}, \quad S_{y}=\sum_{f_{2}=1}^{N_{y}} e^{-i \frac{\pi}{b}\left(n-n^{\prime}\right) d_{y} f_{2}},
$$

where $d_{x}$ and $d_{y}$ are the periods of the photonic crystal along the $x$ and $y$ axes, $N_{x}=\frac{a}{d_{x}}$ and $N_{y}=\frac{b}{d_{y}}$ are the number of cells along the $x$ and $y$ axes, respectively; coordinates of different cells $x_{i}=d_{x} f_{1}, y_{i}=d_{y} f_{2}$ are determined by integers $f_{1}$ and $f_{2}$. To estimate, what values $S_{x}$ can take (the same reasoning is valid for $S_{y}$ ), the above presented expression can be rewritten as follows:

$$
S_{x}=\sum_{f_{1}=1}^{N_{x}} e^{-i \frac{\pi}{a}\left(m-m^{\prime}\right) d_{x} f_{1}}=e^{i \frac{\pi}{2 a}\left(m-m^{\prime}\right)\left(N_{x}-1\right) d_{x}} \frac{\sin \frac{\pi\left(m-m^{\prime}\right) d_{x} N_{x}}{2 a}}{\sin \frac{\pi\left(m-m^{\prime}\right) d_{x}}{2 a}} .
$$

Using (18) for $m-m^{\prime}=0$ one can obtain $S_{x}=N_{x}$. When $m-m^{\prime}=1$, a simple reasoning enables to get what $S_{x}$ is equal to: factor $d_{x} N_{x}=a$ and, hence, the numerator is equal to $\sin \frac{\pi}{2}=1$, while in the denominator $\sin \frac{\pi d_{x}}{2 a} \approx \frac{\pi}{2 N_{x}}$. Therefore, the ratio $\frac{S_{x}\left(m-m^{\prime}=1\right)}{S_{x}\left(m-m^{\prime}=0\right)}=\frac{2}{\pi} \approx 0.6$. With growing 
difference $\left(m-m^{\prime}\right)$, the contribution to the sum of the next terms diminishes provided the following equality is fulfilled:

$$
\frac{\pi\left(m-m^{\prime}\right) d_{x}}{2 a}=\pi \mathrm{P},
$$

where $\mathrm{P}= \pm 1, \pm 2 \ldots$; in these cases the sum $S_{x}=N_{x}$.

Fulfillment of conditions declared by equalities like (19) is equivalent to fulfillment of conditions $k_{x m}-k_{x m^{\prime}}^{\prime}=\tau_{x}$ (i.e., $k_{x m^{\prime}}^{\prime}=k_{x m}-\tau_{x}$ ) and $k_{y n}-k_{y n^{\prime}}^{\prime}=\tau_{y}$ (i.e., $k_{y n^{\prime}}^{\prime}=k_{y n}-\tau_{y}$ ), where $\tau_{x}=\frac{2 \pi}{d_{x}} F$ and $\tau_{y}=\frac{2 \pi}{d_{y}} F^{\prime}$ are $x$ and $y$ components of the reciprocal lattice vector of the photonic crystal, respectively, $F, F^{\prime}=0, \pm 1, \pm 2 \ldots$ Therefore, the major contribution to the sums in the left-hand side of Eq. (13) comes from the amplitudes $C_{m^{\prime} n^{\prime}}^{\lambda^{\prime}}\left(k_{z}^{\prime}, \omega\right) \equiv$ $C^{\lambda^{\prime}}\left(\vec{k}_{\perp m n}-\vec{\tau}_{\perp}, k_{z}-\tau_{z}, \omega\right)=C^{\lambda^{\prime}}\left(\vec{k}_{m n}-\vec{\tau}, \omega\right)$.

Hereafter, when describing electron beam instability, we consider only modes which satisfy the equalities similar to (19). The contribution of other modes is supposed to be suppressed. Thus, the system of Eq. (13) reads as follows:

$$
\left(\vec{k}_{m n}^{2}-\frac{\omega^{2}}{c^{2}}\right) C^{\lambda}\left(\vec{k}_{m n}, \omega\right)-\frac{\omega^{2}}{c^{2}} \sum_{\lambda^{\prime} \tau} \chi_{m n}^{\lambda \lambda^{\prime}}(\vec{\tau}) C^{\lambda^{\prime}}\left(\vec{k}_{m n}-\vec{\tau}, \omega\right)=-\frac{\omega_{L}^{2}\left(k_{m n}^{2} c^{2}-\omega^{2}\right)}{\gamma c^{4}\left(\omega-\vec{k}_{m n} \vec{u}\right)^{2}}\left\{\frac{1}{2 \pi}\left|\int \vec{u} \vec{Y}_{m n}^{\lambda}\left(\vec{k}_{\perp}, k_{z}\right) d^{2} k_{\perp}\right|^{2}\right\} C^{\lambda}\left(\vec{k}_{m n}, \omega\right),
$$

i.e.,

$$
\begin{aligned}
& \left(\vec{k}_{m n}^{2}-\frac{\omega^{2}}{c^{2}}\left[1+\chi_{m n}^{\lambda \lambda}(0)-\frac{\omega_{L}^{2}\left(k_{m n}^{2} c^{2}-\omega^{2}\right)}{\omega^{2} \gamma c^{2}\left(\omega-\vec{k}_{m n} \vec{u}\right)^{2}}\left\{\frac{1}{2 \pi}\left|\int \vec{u} \vec{Y}_{m n}^{\lambda}\left(\vec{k}_{\perp}, k_{z}\right) d^{2} k_{\perp}\right|^{2}\right\}\right]\right) C^{\lambda}\left(\vec{k}_{m n}, \omega\right) \\
& \quad-\frac{\omega^{2}}{c^{2}} \sum_{\lambda^{\prime} \tau} \chi_{m n}^{\lambda \lambda^{\prime}}(\vec{\tau}) C^{\lambda^{\prime}}(\vec{k}-\vec{\tau}, \omega)=0
\end{aligned}
$$

where $\quad \chi_{m n}^{\lambda \lambda^{\prime}}(\tau)=\frac{1}{d_{z}} \int \vec{Y}_{m n}^{\lambda *}\left(\vec{r}_{\perp}, k_{z}\right) \hat{\chi}\left(\vec{r}_{\perp}, \tau_{z}\right) \vec{Y}_{m^{\prime} n^{\prime}}^{\lambda^{\prime}}\left(\vec{r}_{\perp}, k_{z}-\right.$ $\left.\tau_{z}\right) d^{2} r_{\perp}, \quad \hat{\chi}\left(\vec{r}_{\perp}, \tau_{z}\right)=\sum_{x_{i}, y_{i}} \int \hat{\chi}_{\text {cell }}\left(x-x_{i}, y-y_{i}, \zeta\right) e^{-i \tau_{z} \zeta} d \zeta$ $m^{\prime}$ and $n^{\prime}$ are determined by conditions like (19), and $\omega_{L}$ is the Langmuir frequency, $\omega_{L}^{2}=\frac{4 \pi e^{2} \rho_{b 0}}{m}$.

Since this system of equations is homogeneous, for the existence of a nontrivial solution the system determinant must vanish. This condition determines the dispersion equation.

Note that expression $\vec{k}_{m n}-\vec{k}=\vec{\tau}$ is very similar to the Bragg condition and even converts to it, when the transverse dimensions of a waveguide $a$ and $b$ tend to infinity. Actually, when $a, b \rightarrow \infty$ the spectrum of eigenvalues becomes continuous and the set of wave vectors $\vec{k}_{m n}$ converts to wave vector $\vec{k}^{\prime}$ of the wave propagating in the direction determined by Bragg condition $\vec{k}^{\prime}-\vec{k}=\vec{\tau}$, which in more familiar notation reads as

$$
2 d_{g} \sin \theta_{B}=m \lambda_{B},
$$

where $d_{g}$ is the diffraction grating period, $\theta_{B}$ is the Bragg angle, which determines the direction of diffracted wave propagation, $\lambda_{B}$ is the wavelength of the diffracted wave, and $m$ is an integer number. Smooth rotation of diffraction grating with respect to electron velocity at angle $\varphi$ converts $d_{g}$ in (22) to $d_{g} \cos \varphi$, thus making $\lambda_{B}$ and $\theta_{B}$ in (22) slowly changing in a wide range.
The system of Eq. (21) is similar to that describing instability of a beam passing through an infinite crystal $[32,33]$. However, the coefficients appearing in these two systems are differently defined: for an infinite crystal, the wave vectors have continuous spectrum of eigenvalues rather than the discrete spectrum relevant for a bounded photonic crystal. These equations enable one to define dependence $k(\omega)$ for the waves propagating in the crystal. Matching the incident wave packet with the set of waves propagating inside the photonic crystal by the boundary conditions, one can obtain the explicit solutions of the considered equations: the result obtained is formally analogous to that given in [34].

According to (21), the expression within the square brackets acts as dielectric permittivity $\varepsilon$ of the crystal in the case when diffraction can be neglected:

$$
\begin{aligned}
\varepsilon= & n^{2} \\
= & 1+\chi_{m n}^{\lambda \lambda}(0)-\frac{\omega_{L}^{2}\left(k_{m n}^{2} c^{2}-\omega^{2}\right)}{\omega^{2} \gamma c^{2}\left(\omega-\vec{k}_{m n} \vec{u}\right)^{2}} \\
& \times\left\{\frac{1}{2 \pi}\left|\int \vec{u} \vec{Y}_{m n}^{\lambda}\left(\vec{k}_{\perp}, k_{z}\right) d^{2} k_{\perp}\right|^{2}\right\},
\end{aligned}
$$

$n$ is the refractive index i.e., photonic crystal acts as a medium that can be described by a certain refractive index $n$ or the dielectric permittivity $\varepsilon$. The refractive index of the photonic crystal in the absence of the beam $n_{0}$ is 
determined by $n_{0}^{2}=\varepsilon_{0}=1+\chi_{m n}^{\lambda \lambda}(0)$. Two terms contribute to dielectric permittivity (23): scattering of waves by the unit cell of the crystal and scattering of waves by beam electrons. The latter is given by the term proportional to $\omega_{L}^{2}$ and increases when $\omega \rightarrow \vec{k} \vec{u}$.

According to the above analysis the infinite number of modes can be excited by an electron beam moving in a photonic crystal in an arbitrary direction. To distinguish a certain mode one should analyze spectral-angular distribution of radiated photons and select those with certain escape direction and frequency. Depending on location of radiation detector with respect to beam velocity or depending on radiation frequency one can observe photons produced due to the Cherenkov effect or those produced via the effects associated with diffraction. In the case when the detector is tuned to observe photons produced via Cherenkov radiation mechanism only Cherenkov radiation can be considered, while diffracted photons can be neglected. Among the variety one can observe those modes, for which wave vectors $\vec{k}_{m n}$ do not comply Bragg's condition i.e., $\vec{k}_{m n} \neq \vec{k}_{m n}-\vec{\tau}$ for all reciprocal lattice vectors $\vec{\tau}$. When such inequality is valid, the electron beam excites all the modes, but the amplitude of waves arisen due to diffraction appears to be much smaller than the amplitude of the wave with wave vector $\vec{k}_{m n}$. In this case crystal is similar to homogenous matter with dielectric permittivity $\varepsilon_{0}$; when $\varepsilon_{0}>1$ Cherenkov radiation is possible. It is important to note that, though the amplitudes of diffracted waves are small, they, being considered, give the so-called diffraction radiation. Influence of diffraction on beam radiative instability will be analyzed in detail in Sec. III B hereafter. In Sec. III A the radiative instability caused by Cherenkov radiation from the electron beam in a bounded photonic crystal, when waves excited by the beam do not comply Bragg's diffraction conditions, is considered.

\section{A. Electron beam instability in a bounded photonic crystal when waves excited by the beam do not comply Bragg's diffraction conditions}

Let us first consider waves for which Bragg's diffraction conditions are not fulfilled. In this case the sum over $\tau$ in (21) can be dropped, and the conditions for the wave existence are given by putting equal to zero the expression, which is the coefficient at $C^{\lambda}\left(\vec{k}_{m n}, \omega\right)$. This requirement can be written in the form

$$
\begin{aligned}
(\omega & \left.-k_{z} u\right)^{2}\left(k_{m n}^{2}-\frac{\omega^{2}}{c^{2}} n_{0}^{2}\right) \\
= & -\frac{\omega_{L}^{2}\left(k_{m n}^{2} c^{2}-\omega^{2}\right)}{\gamma c^{4}}\left\{\frac{1}{2 \pi}\left|\int \vec{u} \vec{Y}_{m n}^{\lambda}\left(\vec{k}_{\perp}, k_{z}\right) d^{2} k_{\perp}\right|^{2}\right\},
\end{aligned}
$$

therefore

$$
\begin{aligned}
& \left(k_{z}^{2}-\left(\frac{\omega^{2}}{c^{2}} n_{0}^{2}-\varkappa_{m n}^{2}\right)\right)\left(\omega-k_{z} u\right)^{2} \\
& =-\frac{\omega_{L}^{2}\left(k_{m n}^{2} c^{2}-\omega^{2}\right)}{\gamma c^{4}}\left\{\frac{1}{2 \pi}\left|\int \vec{u} \vec{Y}_{m n}^{\lambda}\left(\vec{k}_{\perp}, k_{z}\right) d^{2} k_{\perp}\right|^{2}\right\},
\end{aligned}
$$

where velocity $\vec{u}|| o z$. Equation (24) can be rewritten in the form

$$
\begin{aligned}
\left(k_{z}\right. & \left.-k_{1 z}\right)\left(k_{z}+k_{1 z}\right)\left(k_{z}-k_{2 z}\right)^{2} \\
& =-\frac{\omega_{L}^{2}\left(k_{m n}^{2} c^{2}-\omega^{2}\right)}{\gamma c^{4}}\left\{\frac{1}{2 \pi}\left|\int \vec{u} \vec{Y}_{m n}^{\lambda}\left(\vec{k}_{\perp}, k_{z}\right) d^{2} k_{\perp}\right|^{2}\right\},
\end{aligned}
$$

where $k_{1 z}$ and $k_{2 z}$ are the solutions to the homogeneous equation associated to (24), which issues from (24) by zeroing its right side as follows:

$$
\left(k_{z}-\sqrt{\frac{\omega^{2}}{c^{2}} n_{0}^{2}-\varkappa_{m n}^{2}}\right)\left(k_{z}+\sqrt{\frac{\omega^{2}}{c^{2}} n_{0}^{2}-\varkappa_{m n}^{2}}\right)\left(\omega-k_{z} u\right)^{2}=0,
$$

i.e.,

$$
\begin{gathered}
k_{z}-\sqrt{\frac{\omega^{2}}{c^{2}} n_{0}^{2}-\varkappa_{m n}^{2}}=0, \quad k_{z}+\sqrt{\frac{\omega^{2}}{c^{2}} n_{0}^{2}-\varkappa_{m n}^{2}}=0 \\
\omega-k_{z} u=0 .
\end{gathered}
$$

The roots of Eq. (26) read as follows:

$$
k_{1 z}= \pm \frac{\omega}{c} \sqrt{n_{0}^{2}-\frac{\varkappa_{m n}^{2} c^{2}}{\omega^{2}}}, \quad k_{2 z}=\frac{\omega}{u}
$$

Since $k_{2 z}=\frac{\omega}{u}>0$ in view of the Cherenkov condition given by the third equation in (27), we are concerned with propagation in the photonic crystal of the wave with $k_{1 z}>0$ [i.e., only sign "+" rests in (28)]. In this case in the equation for $k_{z}$, one can take $\left(k_{z}-k_{1 z}\right)\left(k_{z}+k_{1 z}\right) \approx$ $2 k_{1 z}\left(k_{z}-k_{1 z}\right)$ and rewrite Eq. (24) as follows:

$$
\begin{aligned}
& \left(k_{z}-k_{1 z}\right)\left(k_{z}-k_{2 z}\right)^{2} \\
& \qquad=-\frac{\omega_{L}^{2} \omega^{2}\left(n_{0}^{2}-1\right)}{2 k_{1 z} u^{2} \gamma c^{4}}\left\{\frac{1}{2 \pi}\left|\int \vec{u} \vec{Y}_{m n}^{\lambda}\left(\vec{k}_{\perp}, k_{z}\right) d^{2} k_{\perp}\right|^{2}\right\} \\
& \text { i.e., }
\end{aligned}
$$


$A=\frac{\omega_{L}^{2} \omega^{2}\left(n_{0}^{2}-1\right)}{2 k_{1 z} u^{2} \gamma c^{4}}\left\{\frac{1}{2 \pi}\left|\int \vec{u} \vec{Y}_{m n}^{\lambda}\left(\vec{k}_{\perp}, k_{z}\right) d^{2} k_{\perp}\right|^{2}\right\}$,

where $A$ is real and $A>0$ (as to enable the Cherenkov effect, it is necessary to have $\left.n_{0}^{2}>1\right)$.

Thus, $k_{z}$ satisfies cubic Eq. (30). Roots $k_{1 z}$ and $k_{2 z}$ coincide $\left(k_{1 z}=k_{2 z}\right)$, when the particle velocity satisfies the condition as follows:

$$
u=\frac{c}{\sqrt{n_{0}^{2}-\frac{\varkappa_{m n}^{2} c^{2}}{\omega^{2}}}} .
$$

For $k_{1 z}=k_{2 z}$ substitution $\xi=k_{z}-k_{1 z}$ in (30) gives the equation

$$
\xi^{3}=-A
$$

which has three solutions

$$
\xi^{(1)}=-\sqrt[3]{A}, \quad \xi^{(2,3)}=\frac{1}{2}(1 \pm i \sqrt{3}) \sqrt[3]{A} .
$$

The state corresponding to solution $\xi^{(3)}=\frac{1}{2}(1-i \sqrt{3}) \sqrt[3]{A}$ grows with $z$ growing which indicates the presence of instability in a beam [12-14,30,35-37]. In this case the beam instability increment

$$
\delta_{0}^{(3)} \sim \operatorname{Im} k_{z}=\operatorname{Im} \xi^{(3)} \sim \sqrt[3]{\omega_{L}^{2}} \sim \sqrt[3]{\rho_{b 0}}
$$

and the threshold current density (a minimum beam current density required for oscillations to start spontaneously) is determined by the well-known law $j_{\text {thr }} \sim \frac{1}{L^{3}}$ [see Eq. (11.7) in [13] or Eq. (8.63) in [14]].

\section{B. Electron beam radiative instability in a bounded photonic crystal for modes with approximately equal $\vec{k}$ and $\vec{k}+\vec{\tau}$}

Influence of diffraction on beam radiative instability is to be considered hereafter in detail. Let us, thus, consider such a mode excited by the beam, where wave vector $\vec{k}_{m n}$ complies Bragg's diffraction conditions i.e., $\vec{k}_{m n} \simeq \vec{k}_{m n}+\vec{\tau}$. In this case amplitude $C_{m n}(\vec{k}+\vec{\tau})$ can be comparable with amplitude $C_{m n}(\vec{k})$.

Analysis of diffraction of wave $\lambda$, whose electric vector is parallel to the plane $(y, z)$ (a TM-wave) gives

$$
\begin{aligned}
& {\left[k_{m n}^{2}-\frac{\omega^{2}}{c^{2}} \varepsilon\right] C^{\lambda}\left(\vec{k}_{m n}, \omega\right)-\frac{\omega^{2}}{c^{2}} \chi_{m n}^{\lambda \lambda}(-\vec{\tau}) C^{\lambda}\left(\vec{k}_{m n}+\vec{\tau}, \omega\right)=0} \\
& {\left[\left(\vec{k}_{m n}+\vec{\tau}\right)-\frac{\omega^{2}}{c^{2}} \varepsilon_{0}\right] C^{\lambda}\left(\vec{k}_{m n}+\vec{\tau}, \omega\right)} \\
& \quad-\frac{\omega^{2}}{c^{2}} \chi_{m n}^{\lambda \lambda}(\vec{\tau}) C^{\lambda}\left(\vec{k}_{m n}, \omega\right)=0 .
\end{aligned}
$$

Since the term containing $(\omega-(\vec{k}+\vec{\tau}) \vec{u})^{-1}$ is small when $(\omega-\vec{k} \vec{u})$ vanishes, in the second equation it is dropped.

The dispersion equation defining the relation between $k_{z}$ and $\omega$ is obtained by equating to zero the determinant of the system (36) and has a form

$$
\begin{aligned}
& {\left[\left(k_{m n}^{2}-\frac{\omega^{2}}{c^{2}} \varepsilon_{0}\right)\left(\left(\vec{k}_{m n}+\vec{\tau}\right)^{2}-\frac{\omega^{2}}{c^{2}} \varepsilon_{0}\right)-\frac{\omega^{4}}{c^{4}} \chi_{\tau} \chi_{-\tau}\right]\left(\omega-k_{z} u\right)^{2}} \\
& \quad=-\frac{\omega_{L}^{2}}{\gamma c^{4}}\left\{\frac{1}{2 \pi}\left|\int \vec{u} \vec{Y}_{m n}^{\lambda}\left(\vec{k}_{\perp}, k_{z}\right) d^{2} k_{\perp}\right|^{2}\right\}\left(k_{m n}^{2} c^{2}-\omega^{2}\right)\left(\left(\vec{k}_{m n}+\vec{\tau}\right)^{2}-\frac{\omega^{2}}{c^{2}} \varepsilon_{0}\right) .
\end{aligned}
$$

Similar to Sec. III A let us write solutions to the homogeneous equation associated to (37), which issues from (37) by zeroing its right side:

$$
\begin{aligned}
& \left(k_{z}^{2}-\left(\frac{\omega^{2}}{c^{2}} \varepsilon_{0}-\varkappa_{m n}^{2}\right)\right)\left(\left(k_{z}+\tau\right)^{2}-\left(\frac{\omega^{2}}{c^{2}} \varepsilon_{0}-\left(\vec{\varkappa}_{m n}+\vec{\tau}_{\perp}\right)^{2}\right)\right)-\frac{\omega^{4}}{c^{4}} \chi_{\tau} \chi_{-\tau}=0 \\
& \left(k_{z}-\frac{\omega}{u}\right)^{2}=0 .
\end{aligned}
$$

The roots for the system of equations (38) are sought in the vicinity of condition $k_{m n}^{2} \approx\left(\vec{k}_{m n}+\vec{\tau}\right)^{2}$, by substitution $\xi=k_{z}-k_{z 0}$, which gives the expressions as follows:

$$
\begin{aligned}
k_{z} & =k_{z 0}+\xi, \quad k_{z}^{2}=k_{z 0}^{2}+2 k_{z 0} \xi+\xi^{2}, \quad k_{z 0}^{2}=\frac{\omega^{2}}{c^{2}} \varepsilon_{0}-\varkappa_{m n}^{2}, \quad k_{z 0}= \pm \frac{\omega}{c} \sqrt{\varepsilon_{0}-\frac{\varkappa_{m n}^{2} c^{2}}{\omega^{2}},} \\
\left(k_{z}+\tau_{z}\right)^{2} & =\left[\left(k_{z 0}+\tau_{z}\right)+\xi\right]^{2}=\left(k_{z 0}+\tau_{z}\right)^{2}+2\left(k_{z 0}+\tau_{z}\right) \xi+\xi^{2} .
\end{aligned}
$$


Hence, the transformation of expressions is as follows:

$$
\begin{aligned}
& \left(k_{z 0}+\tau_{z}\right)^{2}+\left(\vec{\varkappa}_{m n}+\vec{\tau}_{\perp}\right)^{2}+2\left(k_{z 0}+\tau_{z}\right)+2\left(k_{z 0}+\tau_{z}\right) \xi \\
& \quad+\xi^{2}=\left(\vec{k}_{m n}+\vec{\tau}\right)^{2}+2\left(k_{z 0}+\tau_{z}\right) \xi+\xi^{2}=k_{0 m n}^{2} \\
& \quad+2 \vec{k}_{0 m n} \vec{\tau}+\tau^{2}+2\left(k_{z 0}+\tau_{z}\right) \xi+\xi^{2}
\end{aligned}
$$

enables one to render the first equation in (38) as follows:

$$
2 k_{z 0} \xi\left(2\left(k_{z 0}+\tau_{z}\right) \xi+\left(2 \vec{k}_{0 m n} \vec{\tau}+\tau^{2}\right)\right)-\frac{\omega^{4}}{c^{4}} \chi_{\tau} \chi_{-\tau}=0,
$$

which is equivalent to

$$
4 k_{z 0}\left(k_{z 0}+\tau_{z}\right) \xi^{2}+2 k_{z 0}\left(2 \vec{k}_{0 m n} \vec{\tau}+\tau^{2}\right) \xi-\frac{\omega^{4}}{c^{4}} \chi_{\tau} \chi_{-\tau}=0
$$

Thus, the second-order equation,

$$
\xi^{2}+\frac{\left(2 \vec{k}_{0 m n} \vec{\tau}+\tau^{2}\right)}{\left(k_{z 0}+\tau_{z}\right)} \xi-\frac{\omega^{4}}{c^{4}} \frac{\chi_{\tau} \chi_{-\tau}}{4 k_{z 0}\left(k_{z 0}+\tau_{z}\right)}=0,
$$

enables one to get the following solutions for $\xi$ :

$$
\begin{aligned}
\xi_{1,2}= & -\frac{\left(2 \vec{k}_{0} \vec{\tau}+\tau^{2}\right)}{4\left(k_{z 0}+\tau_{z}\right)} \\
& \pm \sqrt{\left(\frac{2 \vec{k}_{0} \vec{\tau}+\tau^{2}}{4\left(k_{z 0}+\tau_{z}\right)}\right)^{2}+\frac{\omega^{4}}{c^{4}} \frac{\chi_{\tau} \chi_{-\tau}}{4 k_{z 0}\left(k_{z 0}+\tau_{z}\right)}} .
\end{aligned}
$$

When $\left(k_{z 0}+\tau_{z}\right)=-\left|k_{z 0}+\tau_{z}\right|$, the second term in (42) could become equal to zero. At the same time, the second equation in (38) must hold:

$$
\omega-k_{z} u=\omega-k_{z 0} u-\xi u=0 .
$$

Consequently,

$$
\begin{aligned}
\xi_{3} & =\frac{\omega-k_{z 0} u}{u}=\frac{\omega}{u}-k_{z 0}=\frac{\omega}{u}-\frac{\omega}{c} \sqrt{\varepsilon_{0}-\frac{\varkappa_{m n}^{2} c^{2}}{\omega^{2}}} \\
& =\frac{\omega}{u}\left(1-\beta \sqrt{\varepsilon_{0}-\frac{\varkappa_{m n}^{2} c^{2}}{\omega^{2}}}\right) .
\end{aligned}
$$

If $\varepsilon_{0}<1$, then $\xi_{3}=\frac{\omega}{u}-k_{z 0}>0$, Let solutions $\xi_{1}$ and $\xi_{2}$ coincide $\left(\xi_{1}=\xi_{2}\right)$. This is possible at the point, where

$$
\frac{2 \vec{k}_{0} \vec{\tau}+\tau^{2}}{4\left(k_{z 0}+\tau_{z}\right)}= \pm \frac{\omega^{2}}{c^{2}} \frac{\sqrt{\chi_{\tau} \chi_{-\tau}}}{\sqrt{4 k_{z 0}\left|k_{z 0}+\tau_{z}\right|}},
$$

here $k_{z 0}+\tau_{z}<0$, and the following equality is fulfilled:

$$
\frac{\omega}{u}-k_{z 0}=\mp \frac{\omega^{2}}{c^{2}} \frac{\sqrt{\chi_{\tau} \chi_{-\tau}}}{\sqrt{4 k_{z 0}\left|k_{z 0}+\tau_{z}\right|}},
$$

i.e.,

$$
\frac{\omega}{u}=k_{z 0} \mp \frac{\omega^{2}}{c^{2}} \frac{\sqrt{\chi_{\tau} \chi_{-\tau}}}{\sqrt{4 k_{z 0}\left|k_{z 0}+\tau_{z}\right|}},
$$

where $k_{z 0}=\frac{\omega}{c} \sqrt{\varepsilon_{0}-\frac{\chi_{m n}^{2} c^{2}}{\omega^{2}}}$.

When $\varepsilon_{0}<1$, the following ratio is valid $\frac{\omega}{u}>k_{z 0}$ (since $u<c$ ), and for solution $\frac{\omega}{u}=k_{z 0}-\frac{\omega^{2}}{c^{2}} \frac{\sqrt{\chi_{\tau} \chi_{-\tau}}}{\sqrt{4 k_{z 0}\left|k_{z 0}+\tau_{z}\right|}}$ (44) the Cherenkov condition is not fulfilled.

Now let us consider the solution $\frac{\omega}{u}=k_{z 0}+\frac{\omega^{2}}{c^{2}} \frac{\sqrt{x_{x} \chi_{-\tau}}}{\sqrt{4 k_{z 0}\left|k_{z 0}+\tau_{z}\right|}}$. At $\tau_{z}<0$ the difference $k_{z 0}+\tau_{z}$ can be reduced to make the sum on the right side equal to $\frac{\omega}{u}$, thus providing equality of all four dispersion equation roots.

Note that for backward diffraction, which is conventional for frequently used one-dimensional generators with a corrugated metal waveguide (traveling-wave tube, backward-wave oscillator), such coincidence of roots is impossible. Indeed, suppose the solutions $\xi_{1}$ and $\xi_{2}$ coincide in case of backward Bragg diffraction $\left(\left|\tau_{z}\right| \approx 2 k_{z 0}\right.$, $\left.\tau_{z}<0\right)$. Then by substituting the expressions for

$$
k_{z 0}=\frac{\omega}{c} \sqrt{\varepsilon_{0}-\frac{\chi_{m n}^{2} c^{2}}{\omega^{2}}} \text { and } \varepsilon_{0}=n_{0}^{2}=1+\chi_{m n}^{\lambda \lambda}(0)
$$

and retaining the first-order infinitesimal terms, the relation

$$
\frac{\omega}{u} \approx k_{z 0}+\frac{\omega^{2}}{c^{2}} \frac{\left|\chi_{\tau}\right|}{2 k_{z 0}}
$$

can be reduced to the form

$$
\frac{\omega}{u} \approx \frac{\omega}{c}\left(1-\frac{\left|\chi_{m n}^{\lambda \lambda}(0)\right|}{2}-\frac{\chi_{m n}^{2} c^{2}}{2 \omega^{2}}+\frac{\omega}{c} \frac{\left|\chi_{\tau}\right|}{2}\right)<\frac{\omega}{u},
$$

i.e., the equality does not hold and the fourfold degeneracy is impossible. Only the case of threefold degeneration considered in Sec. III A is possible. However, if $\varepsilon_{0}>1$ and is appreciably large, then in a one-dimensional case, the fourfold degeneracy of roots is also possible in a finite photonic crystal.

Thus, the left side of Eq. (37) has four solutions $\left(\xi_{1}, \xi_{2}\right.$, and a double degenerated $\xi_{3}$ ). Hence, Eq. (37) can be written as follows:

$$
\left(\xi-\xi_{1}\right)\left(\xi-\xi_{2}\right)\left(\xi-\xi_{3}\right)^{2}=-B,
$$

where $\xi_{1,2}$ and $\xi_{3}$ are defined by (42) and (43), respectively, $B$ is real, $B>0$, 


$$
\begin{aligned}
B= & \frac{\omega_{L}^{2}}{4 k_{z 0}\left(k_{z 0}+\tau_{z}\right) u^{2} \gamma c^{4}}\left\{\frac{1}{2 \pi}\left|\int \vec{u} \vec{Y}_{m n}^{\lambda}\left(\vec{k}_{\perp}, k_{z}\right) d^{2} k_{\perp}\right|^{2}\right\} \\
& \times\left(k_{m n}^{2} c^{2}-\omega^{2}\right)\left(\left(\vec{k}_{m n}+\vec{\tau}\right)^{2}-\frac{\omega^{2}}{c^{2}} \varepsilon_{0}\right) .
\end{aligned}
$$

If suppose $\xi_{1}=\xi_{2}=\xi_{3}$, then Eq. (45) converts to

$$
\left(\xi-\xi_{1}\right)^{4}=-B, \quad \text { i.e., } \xi-\xi_{1}=\sqrt[4]{-B},
$$

thus we define four solutions as follows:

$$
\begin{array}{ll}
\xi_{1}^{(1)}=\frac{1}{\sqrt{2}}(1+\imath) \sqrt[4]{B}, \quad \xi_{1}^{(2)}=\frac{1}{\sqrt{2}}(-1+\imath) \sqrt[4]{B} \\
\xi_{1}^{(3)}=-\frac{1}{\sqrt{2}}(1+\imath) \sqrt[4]{B}, \quad \xi_{1}^{(4)}=-\frac{1}{\sqrt{2}}(-1+\imath) \sqrt[4]{B}
\end{array}
$$

The above results can be illustrated as follows. In general case frequency $\omega(\vec{k})$ of a photon moving in a spatially periodic media (crystal) is a periodic function of reciprocal lattice vector $\tau$, i.e., $\omega(\vec{k})=\omega(\vec{k}+\tau)$. Such dependence is conventionally illustrated by a dispersion curve (see, for example, [38] Chapter 8), which is schematically shown in Fig. 1. A dispersion relation usually describes the frequency-dependent propagation characteristics of a one-dimensional periodic system (e.g. corrugated waveguide) used for radiation excitation in the traveling wave tube or backward wave oscillator [12-14,35-38]. In the case under consideration the similar approach is valid for two- or three-dimensional photonic crystals placed inside a waveguide of rectangular cross section, when electron beam moves along the waveguide axis and diffraction occurs for the reciprocal lattice vector, which is antiparallel to beam velocity $\vec{\tau}=\left(\tau_{x}=0, \tau_{y}=0, \tau_{z}\right) \uparrow \downarrow \vec{u}$. The frequency of radiation produced by the electron beam is determined by interception of dispersion curve $\omega\left(k_{z}\right)$ with the beam line $\left(\omega-k_{z} u\right)=0$ (see Fig. 1) which means synchronism of wave and beam electrons. Interceptions can occur at different values of $v_{g r}=\frac{d \omega}{d k}$ : at $v_{g r}>0$ wave travels forward along beam velocity [such a situation is typical for traveling wave tubes (left plot in Fig. 1)]; wave with $v_{g r}<0$ moves backward enabling generation of backward wave oscillators [12-14,35-39] (center plot in Fig. 1). Degeneration of roots of the dispersion equation puts the synchronism point to the $\pi$ point of the dispersion curve (right plot in Fig. 1). In this point amplitudes of direct and diffracted waves are comparable to each other.

Solutions $\xi_{1}^{(3)}$ and $\xi_{1}^{(4)}$ have negative imaginary parts and cause exponential growth of field amplitude, thus they are responsible for beam instability. Increment of beam instability in case of four roots degeneracy reads as follows:

$$
\delta_{0}^{(4)} \sim \operatorname{Im} k_{z}=\operatorname{Im} \xi_{1}^{(3,4)} \sim \sqrt[4]{\omega_{L}} \sim \sqrt[4]{\rho_{b 0}} .
$$

To compare increments $\delta_{0}^{(3)}$ and $\delta_{0}^{(4)}$ let us consider the case when solutions $\xi_{1} \neq \xi_{2}$ and $\xi_{1}-\xi_{2} \gg \chi_{\tau}$ and study (45) for $\xi \rightarrow \xi_{1}$, thus reducing the order of the equation:

$$
\left(\xi-\xi_{1}\right)\left(\xi-\xi_{3}\right)^{2}=-\frac{B}{\xi_{1}-\xi_{2}} .
$$

The obtained equation is conformable to (30). Hence, the ratio $\frac{\delta_{0}^{(4)}}{\delta_{0}^{(3)}}$ can be expressed as

$$
\frac{\delta_{0}^{(4)}}{\delta_{0}^{(3)}}=\sqrt{\frac{2}{3}} \sqrt[3]{\xi_{1}-\xi_{2}} \frac{1}{\sqrt[B]{12}} .
$$

For the sake of evaluations (46) can be replaced by the approximate expression for $B$ as follows:

$$
B \approx \frac{\omega_{L}^{2}}{\omega^{2} \gamma} k_{0}^{4} \chi_{0}^{4}
$$

hence, evaluation for $\delta_{0}^{(4)}$ and $\delta_{0}^{(3)}$ reads
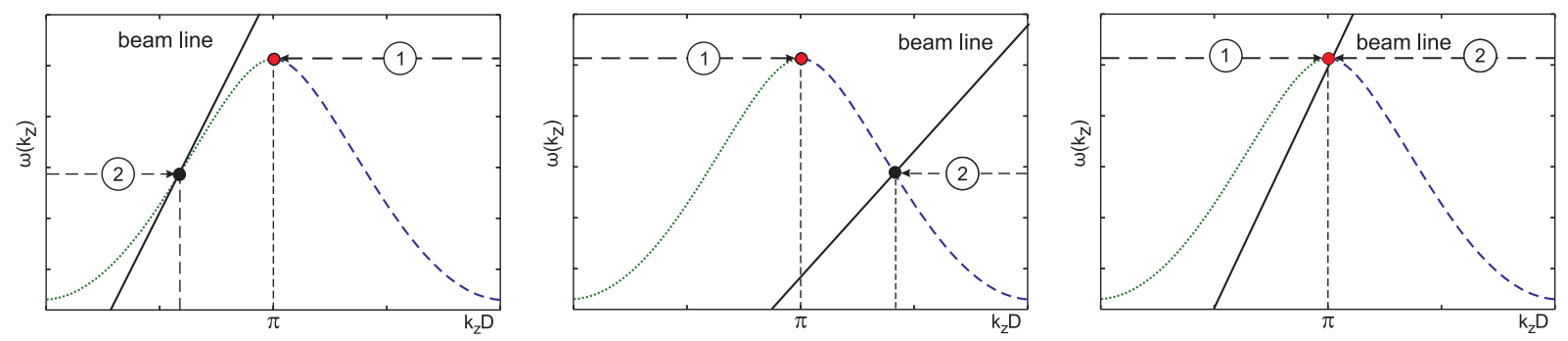

FIG. 1. Schematic drawing of dispersion curve $\omega\left(k_{z}\right)$. Point marked 1 corresponds to $v_{g r}=0$ ( $\pi$-point), it matches $k_{z} D=\pi$, where $D$ is the period of periodic structure. Points marked 2 correspond to beam-wave synchronism points. The left plot is for traveling wave tube (TWT), center plot corresponds to backward wave oscillator (BWO) case; the right plot describes interaction in the $\pi$ point. 


$$
\begin{aligned}
\delta_{0}^{(4)} & \approx \frac{1}{\sqrt{2}} \sqrt[4]{k_{0}^{4} \chi_{0}^{4} \frac{\omega_{L}^{2}}{\omega^{2} \gamma}}=\frac{1}{\sqrt{2}} \frac{k_{0} \chi_{0}}{\sqrt[4]{\gamma}} \sqrt{\frac{\omega_{L}}{\omega}}, \\
\delta_{0}^{(3)} & \approx \frac{\sqrt{3}}{2} \sqrt[3]{k_{0}^{3} \chi_{0}^{3} \frac{\omega_{L}^{2}}{\omega^{2} \gamma}}=\frac{\sqrt{3}}{\sqrt{2}} \delta_{0}^{(4)} \gamma^{-\frac{1}{12}} \sqrt[6]{\frac{\omega_{L}}{\omega}}
\end{aligned}
$$

and ratio $\frac{\delta_{0}^{(4)}}{\delta_{0}^{(3)}}$ can be evaluated by

$$
\frac{\delta_{0}^{(4)}}{\delta_{0}^{(3)}}=\sqrt{\frac{2}{3}} \gamma^{\frac{1}{12}} \sqrt[6]{\frac{\omega}{\omega_{L}}} .
$$

For terahertz range even for a beam with current density $j \sim 10^{8} \mathrm{~A} / \mathrm{cm}^{2}\left(\omega_{L} \sim 10^{8} \mathrm{~Hz}\right)$ expression (52) gives $\frac{\delta_{0}^{(4)}}{\delta_{0}^{(3)}} \gg 1$.

The typical length, at which instability develops, is inverse to the increment value, therefore, along with (52) the following relations are also valid:

$$
\begin{aligned}
& \frac{L^{(3)}}{L^{(4)}}=\frac{\delta_{0}^{(4)}}{\delta_{0}^{(3)}}=\sqrt{\frac{2}{3}} \gamma^{\frac{1}{12}} \sqrt[6]{\frac{\omega}{\omega_{L}}} \\
& L^{(4)}=\frac{1}{\delta_{0}^{(4)}}=\sqrt{2} \frac{\sqrt[4]{\gamma}}{k_{0} \chi_{0}} \sqrt{\frac{\omega}{\omega_{L}}}, \\
& L^{(3)}=\frac{1}{\delta_{0}^{(3)}}=\frac{2}{\sqrt{3}} \frac{\sqrt[3]{\gamma}}{k_{0} \chi_{0}} \sqrt[3]{\frac{\omega^{2}}{\omega_{L}^{2}}},
\end{aligned}
$$

thus providing the following evaluation for the lengths' ratio, at which instability develops, $\frac{L^{(3)}}{L^{(4)}} \gg 1$. Therefore, generation in the vicinity of the $\pi$ point (or, equally, in the case when amplitudes of direct and diffracted waves approximate to each other) gives a advantage of shorter length, at which instability develops [15].

Let us analyze what values $L^{(3)}$ and $L^{(4)}$ can possess at typical parameters of modern accelerators and radiation frequency 1 terahertz $\left(\lambda=3 \times 10^{-2} \mathrm{~cm}\right)$. Suppose electron beam energy is $8 \mathrm{MeV}$, bunch transverse size $250 \mu \mathrm{m} \times$ $250 \mu \mathrm{m}$, and bunch charge $25 \mathrm{pC}$ [40]. Let us also consider a photonic crystal formed by parallel metallic wires, which are parallel to the waveguide boundary $(y, z)$. According to $[5,41]$ for such a photonic crystal with wires of $10^{-3} \mathrm{~cm}$ diameter spaced $6 \times 10^{-2} \mathrm{~cm}$ the susceptibility value is $\chi_{0} \approx 3 \times 10^{-1}$. For selected parameters, according to (54), length $L^{(4)} \approx 70 \mathrm{~cm}$, while $L^{(3)}$ is more than 10 times larger $\left(L^{(3)} \approx 900 \mathrm{~cm}\right.$ ). Increase of electron beam current density could make length $L^{(4)}$ even smaller. The same could be provided by increased susceptibility value, but for $\chi_{0}>1$ detailed numerical analysis is necessary [42].

The threshold generation conditions, i.e., the values of the electron current and other parameters of the beam, at which radiation begins to exceed the losses, can be obtained by solving the boundary-value problem similar to how it was made in [16]. For instance, the expression for the generation threshold under the conditions of two-wave diffraction in the case of cold beam reads

$$
\begin{aligned}
& \frac{1}{4 \gamma} \frac{4 \pi e^{2}}{\omega^{2} m} \frac{j_{\text {thr }}}{u}\left|\frac{1}{u} \int \vec{u} \vec{Y}_{m n}^{\lambda}\left(\vec{k}_{\perp}, k_{z}\right) d^{2} k_{\perp}\right|^{2} f(y) \\
& \quad=16\left(\frac{\gamma_{0} c}{\vec{u} \vec{n}}\right)^{3} \frac{\beta_{1}}{k^{5} \chi_{\tau}^{2} L^{5}}
\end{aligned}
$$

where $\vec{n}$ is the unit normal vector to the crystal surface (directed toward the interior); $L$ is the crystal thickness; $\chi_{0}$ and $\chi_{\tau}$ are the Fourier expansion coefficients of the crystal dielectric susceptibility; $\beta_{1}=\gamma_{1} / \gamma_{0}$ is the diffraction asymmetry factor; $\beta_{1}=\frac{\gamma_{1}}{\gamma_{0}}=\frac{\vec{n}(\vec{k}+\vec{\tau})}{\vec{n} \vec{k}}, \gamma_{0}$ and $\gamma_{1}$ are the cosines of the angles between the normal vector $\vec{n}$ and the wave vectors of the transmitted $\vec{k}$ and diffracted $\vec{k}+\vec{\tau}$ waves, respectively; the subscript $\perp$ denotes the projection of the vector on the plane perpendicular to $\vec{u} ; f(y)$ is the spectral function depending on detuning from the synchronism conditions defined in [16]:

$$
f(y)=\sin y \frac{(2 y+\pi n) \sin y-y(y+\pi n) \cos y}{y^{3}(y+\pi n)^{3}},
$$

where $y=\frac{k \operatorname{Re}\left(\xi_{2}\right) L}{2}$ and $\xi_{2}$ is the root of the dispersion equation in the absence of the electron beam. Expression (54) provides for $j_{\text {thr }}$ the dependance on the crystal length $L$ as follows: $j_{\text {thr }} \sim \frac{\beta_{1}}{L^{5}}$, which is different from that cited in Sec. III A and $[13,14]$.

The analysis $[9,16,17]$ shows that with increasing the number of diffracted waves, the law established in $[15,43,44]$ is still valid: the instability increment appears to be proportional to $\rho_{b 0}^{\frac{1}{s+3}}$, where $s$ is the number of waves emerging through diffraction. As a result, the abrupt decrease in the threshold generation current also remains in this case (the threshold generation current $j_{\text {thr }} \sim$ $\frac{1}{(k L)^{3}\left(k \chi_{\tau} L\right)^{2 s}}$, where $L$ is the length of the interaction area).

\section{CONCLUSION}

Combining the photonic crystal-based structures with vacuum electronic devices opens the way for creation of a family of radiation sources: volume FELs, photonic BWOs, etc.

The dispersion equations describing electron beam instability in a bounded photonic crystal are obtained for two cases: the conventionally considered case, when diffraction is suppressed, and the case of direct and diffracted waves having almost equal amplitudes. The instability law is shown to be responsible for increase of increment of instability and decrease of length, at which instability develops, for the case when amplitude of 
diffracted wave is comparable with that of the direct one that happens in the vicinity of the $\pi$ point of dispersion curve. Such an instability law enables application of photonic crystals for development of $\mathrm{THz}$ sources at electron beam current densities available at modern accelerators.

Some beneficial options are additionally available; namely, use for radiation generation of multiple either pencil-like or sheet electron beams instead of single annular or sheet one and, thus, establishing the beam-wave interaction within the whole crystal cross section and increasing the efficiency of the radiation source.

\section{APPENDIX: DETAILED CONVERSION FROM MAXWELL EQUATIONS TO DISPERSION EQUATION}

Let us thoroughly consider how Eq. (7) can be obtained from (6). From Maxwell Eq. (2) it follows that

$\operatorname{div} \vec{D}(\vec{r}, t)=\operatorname{div} \vec{E}(\vec{r}, t)+\sum_{i k} \frac{\partial}{\partial x_{i}}\left[\chi_{i k}(\vec{r}) E_{k}(\vec{r})\right]=4 \pi \rho(\vec{r}, t)$

i.e.,

$$
\operatorname{div} \vec{E}(\vec{r}, t)=4 \pi \rho(\vec{r}, t)-\sum_{i, k} \frac{\partial}{\partial x_{i}}\left(\chi_{i k} E_{k}\right)
$$

therefore

$$
\vec{\nabla}(\vec{\nabla} \vec{E}(\vec{r}, t))=4 \pi \vec{\nabla} \rho(\vec{r}, t)-\vec{\nabla}\left(\sum_{i, k} \frac{\partial}{\partial x_{i}}\left(\chi_{i k}(\vec{r}) E_{k}(\vec{r})\right)\right)
$$

As a result Eq. (6) can be rewritten as follows:

$$
\begin{gathered}
\Delta \vec{E}(\vec{r}, t)+\vec{\nabla}\left(\sum_{i k} \frac{\partial}{\partial x_{i}}\left(\chi_{i k}(\vec{r}) E_{k}(\vec{r})\right)\right) \\
-\frac{1}{c^{2}} \frac{\partial^{2}}{\partial t^{2}} \int \hat{\varepsilon}\left(\vec{r}, t-t^{\prime}\right) \vec{E}\left(\vec{r}, t^{\prime}\right) d t \\
=\frac{4 \pi}{c^{2}} \frac{\partial \vec{j}(\vec{r}, t)}{\partial t}+4 \pi \vec{\nabla} \rho(\vec{r}, t) .
\end{gathered}
$$

The system of Eqs. (5)-(7) enables studying radiation produced by an electron beam in a photonic crystal and getting the instability increments. Beam current and density presenting in Eqs. (2)-(6) are complicated functions of $\vec{E}(\vec{r}, t)$. The initial stage of radiation process ("exponentially growing-wave stage of interaction" [35], Sec. 4.2), accompanied by arising the radiative instability, is commonly referred to as the small-signal regime (linear regime), when beam current and density perturbations are the linear functions of $\vec{E}$ [12-14,30,35-39].

Therefore, beam current and density have the form $\vec{j}=\vec{j}_{0}+\delta \vec{j}$ and $\rho=\rho_{0}+\delta \rho$, where $\delta \vec{j}=\delta \vec{j}(\vec{r}, t)$ and $\delta \rho=\delta \rho(\vec{r}, t)$ are linear functions of $\vec{E}$. Thus, the following equation is valid:

$$
\begin{gathered}
\Delta \vec{E}(\vec{r}, t)+\vec{\nabla}\left(\frac{\partial}{\partial x_{i}}\left(\chi_{i k}(\vec{r}) E_{k}(\vec{r})\right)\right)-4 \pi\left(\frac{1}{c^{2}} \frac{\partial \delta \vec{j}}{\partial t}+\vec{\nabla} \delta \rho\right) \\
-\frac{1}{c^{2}} \frac{\partial^{2}}{\partial t^{2}} \int \hat{\varepsilon}\left(\vec{r}, t-t^{\prime}\right) \vec{E}\left(\vec{r}, t^{\prime}\right) d t^{\prime}=\frac{4 \pi}{c^{2}} \frac{\partial \overrightarrow{j_{0}}}{\partial t}+4 \pi \vec{\nabla} \rho_{0},
\end{gathered}
$$

summation over repeated indices is implied, $\vec{j}_{0}=\vec{j}_{0}(\vec{r}, t)$ and $\rho_{0}=\rho_{0}(\vec{r}, t)$ are the beam current and density unperturbed by field.

Recall that in the case under consideration the susceptibility tensor of a photonic crystal is a periodic function of coordinate $\vec{r}$ and reads sa follows: $\hat{\chi}(\vec{r})=\sum_{i} \hat{\chi}_{\text {cell }}\left(\vec{r}-\vec{r}_{i}\right)=$ $\sum_{\vec{\tau}} \hat{\chi}_{\vec{\tau}} e^{i \vec{\tau} \vec{r}}$.

Let us now consider a practically important case, when all the elements $\chi_{i k}$ of tensor $\hat{\chi}$ are much smaller as compared to the unity $\left|\chi_{i k}\right| \ll 1$. Such a condition is always valid in the $\mathrm{x}$-ray range. In many practically important cases it is also applicable for microwave, terahertz and optical ranges. For example, made of periodically strained wires photonic crystals, which are used for generation in microwave range, typically have effective susceptibilities $\chi_{i k} \lesssim 10^{-1}-10^{-2}[5,6,17-19]$.

For further consideration let us make Fourier transform of Eq. (A5), thus getting

$$
\begin{aligned}
& \Delta \vec{E}(\vec{r}, \omega)+\vec{\nabla}\left(\frac{\partial}{x_{i}} \chi_{i k}(\vec{r}) E_{k}(\vec{r}, \omega)\right) \\
& \quad+4 \pi\left(\frac{i \omega}{c^{2}} \delta j(\vec{r}, \omega)-\vec{\nabla} \delta \rho(\vec{r}, \omega)\right)+\frac{\omega^{2}}{c^{2}} \vec{E}(\vec{r}, \omega) \\
& \quad+\frac{\omega^{2}}{c^{2}} \hat{\chi}(\vec{r}) \vec{E}(\vec{r}, \omega)=-\frac{4 \pi i \omega}{c^{2}} j_{0}(\vec{r}, \omega)+4 \pi \vec{\nabla} \rho_{0}(\vec{r}, \omega),
\end{aligned}
$$

where beam current and density are presented in approximation linear over field $\vec{E}$ as $\vec{j}=\vec{j}_{0}+\delta \vec{j}$ and $\rho=\rho_{0}+\delta \rho$, beam current and density perturbations are the linear functions of $\vec{E}$. This Eq. (A6) is equivalent to the linear system of Maxwell equations including external sources. The general solution of this system, which describes the process of radiation and radiative instability, is given by the sum of two solutions, namely, the fundamental solution to the associated homogeneous differential equation [Eq. (A6) with zero right side] and a particular solution to a nonhomogeneous differential equation, which 
describes the process of radiation. The particular solution to a nonhomogeneous differential equation can be presented by the transverse Green function [29]. This function is to be derived from solutions to the associated homogeneous differential equation. Consider first the associated to (A6) homogeneous differential equation, which reads as follows:

$$
\begin{aligned}
\Delta \vec{E}(\vec{r}, \omega)+\frac{\omega^{2}}{c^{2}} \vec{E}(\vec{r}, \omega)+\vec{\nabla}\left(\frac{\partial}{\partial x_{i}}\left(\chi_{i k}(\vec{r}) E_{k}(\vec{r}, \omega)\right)\right) \\
\quad+\frac{\omega^{2}}{c^{2}} \hat{\chi}(\vec{r}) \vec{E}(\vec{r}, \omega)+4 \pi\left(\frac{i \omega}{c^{2}} \delta \vec{j}(\vec{r}, \omega)-\vec{\nabla} \delta \rho(\vec{r}, \omega)\right)=0 .
\end{aligned}
$$

Fourier transform over coordinates $\vec{E}(\vec{r}, \omega)=$ $\frac{1}{(2 \pi)^{3}} \int \vec{E}(\vec{k}, \omega) e^{i \vec{k} \vec{r}} d^{3} k$ applied to (A7) gives

$$
\begin{aligned}
& -k^{2} \vec{E}(\vec{k}, \omega)+\frac{\omega^{2}}{c^{2}} \vec{E}(\vec{k}, \omega) \\
& \left.-\sum_{\tau} \vec{k}\left(k_{i} \chi_{i k}^{\tau} E_{k}(\vec{k}-\vec{\tau})\right\}\right)+\frac{\omega^{2}}{c^{2}} \sum_{\tau} \hat{\chi}_{\tau} \vec{E}(\vec{k}-\vec{\tau}) \\
& +4 \pi\left(\frac{i \omega}{c^{2}} \delta \vec{j}(\vec{k}, \omega)-i \vec{k} \delta \rho(\vec{k}, \omega)\right)=0 .
\end{aligned}
$$

The equation similar to (A8) can be written for the component $\vec{E}(\vec{k}-\vec{\tau})$ :

$$
\begin{aligned}
& -(\vec{k}-\vec{\tau})^{2} \vec{E}(\vec{k}-\vec{\tau}, \omega)+\frac{\omega^{2}}{c^{2}} \vec{E}(\vec{k}-\vec{\tau}, \omega) \\
& -\quad \sum_{\tau^{\prime}}(\vec{k}-\vec{\tau})\left[(\vec{k}-\vec{\tau}) i \chi_{i k}^{\tau^{\prime}} E_{k}\left(\vec{k}-\vec{\tau}-\vec{\tau}^{\prime}\right)\right] \\
& +\frac{\omega^{2}}{c^{2}} \sum_{\tau^{\prime}} \hat{\chi}_{\tau^{\prime}} \vec{E}\left(\vec{k}-\vec{\tau}-\vec{\tau}^{\prime}\right)+4 \pi\left(\frac{i \omega}{c^{2}} \delta \vec{j}(\vec{k}-\vec{\tau}, \omega)\right. \\
& -i(\vec{k}-\vec{\tau}) \delta \rho(\vec{k}-\vec{\tau}, \omega))=0 .
\end{aligned}
$$

The system has a solution, if its determinant is zero. This condition produces the dispersion equation, which establishes the relation between wave vector $\vec{k}$ and frequency $\omega$, thus enabling to find either $\vec{k}$ as a function of $\omega[\vec{k}=\vec{k}(\omega)]$ or $\omega$ as a function of $\vec{k}[\omega=\omega(\vec{k})]$.

Vector $\vec{E}$ possesses two polarizations: transverse polarization, for which $\vec{E}_{\perp}(\vec{k}), \vec{E}_{\perp}(\vec{k}-\vec{\tau})$ are orthogonal to wave vectors $\vec{k}$ and $(\vec{k}-\vec{\tau})$, and a longitudinal one, for which $\vec{E}_{\|}(\vec{k}), \vec{E}_{\|}(\vec{k}-\vec{\tau})$ are directed along wave vectors $\vec{k}$ and $(\vec{k}-\vec{\tau})$. The dynamical theory of diffraction [45], which describes the interaction of waves with a regular lattice, demonstrates that for a crystal with components of susceptibility tensor $\left|\chi_{i k}\right| \ll 1$, the following relation is correct: Components of vector $\vec{E}$, which are orthogonal to photon wave vector $\vec{k}$, and those orthogonal to wave vector $(\vec{k}-\vec{\tau})$ are much greater than the corresponding longitudinal components $\left(\vec{E}_{\perp}(\vec{k}), \quad \vec{E}_{\perp}(\vec{k}-\vec{\tau}) \gg \vec{E}_{\|}(\vec{k})\right.$, $\left.\vec{E}_{\|}(\vec{k}-\vec{\tau})\right)[46,47]$. A similar treatment can be applied to any problem considering wave interaction with a media with spatially periodic susceptibility, whose components are smaller than unity. Due to this relation the third term in Eqs. (A8) and (A9) can be neglected $[46,47]$.

When deriving (A7), which describes propagation of electromagnetic waves in the photonic crystal possessing $\left|\chi_{i k}\right| \ll 1$, the same reason allows to set aside the abovementioned term. All the solutions to the inhomogeneous equation are expressed via solutions to the associated homogeneous Eqs. (A8) and (A9).

To avoid ambiguity it is important to mention that radiative instability arises in conditions of coupling transverse electromagnetic waves and the longitudinal wave caused by longitudinal plasma oscillations of electron beam, which is significant for deriving expressions $\delta \vec{j}$ and $\delta \rho$ in equations above (see in details [12-14,35-39]).

[1] H. A. Hafez, X. Chai, A. Ibrahim, S. Mondal, D. Ferachou, $\mathrm{X}$. Ropagnol, and T. Ozaki, Intense terahertz radiation and their applications, J. Opt. 18, 093004 (2016).

[2] R. A. Lewis, A review of terahertz sources, J. Phys. D 47, 374001 (2014).

[3] V. L. Bratman, A. G. Litvak, and E. V. Suvorov, Mastering the terahertz domain: Sources and applications, Phys. Usp. 54, 837 (2011).

[4] K. Lekomtsev, A. Aryshev, M. Shevelev, N. Terunuma, J. Urakawa, A. Ponomarenko, and A. Tishchenko, Experimental investigation of $\mathrm{THz}$ Smith-Purcell radiation from composite corrugated capillary, in Proceedings of IPAC2016 (Bexco, Busan, Korea, 2016), p. 1861, https://accelconf.web.cern.ch/AccelConf/ipac2016/html/ auth3976.htm.

[5] V. G. Baryshevsky and A. A. Gurinovich, Spontaneous and induced parametric and Smith-Purcell radiation from electrons moving in a photonic crystal built from the metallic threads, Nucl. Instrum. Methods Phys. Res., Sect. B 252, 92 (2006).

[6] V. G. Baryshevsky, N. A. Belous, A. A. Gurinovich, V. A. Evdokimov, P. V. Molchanov, A. V. Oskin, and P. F. Safronov, Experimental study of a volume free electron laser with a "grid" resonator, 28th International Free Electron Laser Conference (FEL 2006) (BESSY, Berlin, 2006), TUPPH012, p. 331, http://accelconf.web.cern.ch/ AccelConf/f06/.

[7] V. Baryshevsky, K. Batrakov, A. Gurinovich, I. Ilienko, A. Lobko, V. Moroz, P. Sofronov, and V. Stolyarsky, First lasing of a volume FEL (VFEL) at a wavelength range $\lambda \sim 4-6 \mathrm{~mm}$, Nucl. Instrum. Methods Phys. Res., Sect. A 483, 21 (2002). 
[8] V. G. Baryshevsky, K. G. Batrakov, V. A. Evdokimov, A. A. Gurinovich, A. S. Lobko, P. V. Molchanov, P. F. Safronov, and V. I. Stolyarsky, Experimental observation of radiation frequency tuning in "OLSE-10" prototype of volume free electron laser, Nucl. Instrum. Methods Phys. Res., Sect. B 252, 86 (2006).

[9] V. G. Baryshevsky, Volume free electron lasers, Nucl. Instrum. Methods Phys. Res., Sect. A 445, 281 (2000).

[10] V. G. Baryshevsky and A. A. Gurinovitch, Hybrid systems with virtual cathode for high power microwaves generation, arXiv:0903.0300v1.

[11] R. L. Ewing, J. S. Cetnar, B. Jogai, C. L. Devlin, and A. M. Ferendeci, Frequency adaptable maser source, in Proceedings of National Aerospace and Electronics Conference (NAECON2015) (Dayton, Ohio, USA), https:// www.researchgate.net/publication/304417631_Frequency_ adaptable_maser_source.

[12] R. B. Miller, An Introduction to the Physics of Intense Charged Particle Beams (Plenum Press, New York, 1982).

[13] V. L. Granatstein and I. Alexeff, High-Power Microwave Sources (Artech House, Boston, 1987).

[14] S. E. Tsimring, Electron Beams and Microwave Vacuum Electronics, Wiley Series in Microwave and Optical Engineering (Wiley, New York, 2006).

[15] V. G. Baryshevsky and I. D. Feranchuk, Parametric bunch instability of electrons in crystals, Phys. Lett. 102A, 141 (1984).

[16] V. G. Baryshevsky, High power microwave and optical volume free electron lasers (VFELs), arXiv:1211.4769v1.

[17] V. G. Baryshevsky, Spontaneous and induced radiation by electrons/positrons in natural and photonic crystals. Volume free electron lasers (VFELs): From microwave and optical to x-ray range, Nucl. Instrum. Methods Phys. Res., Sect. B 355, 17 (2015).

[18] V. G. Baryshevsky and A. A. Gurinovich, Electrodynamical properties of a volume free electron laser with a "grid" resonator, in 28th International Free Electron Laser Conference (FEL 2006) (BESSY, Berlin, 2006), TUPPH013, pp. 335-339, http://accelconf.web.cern.ch/ AccelConf/f06/.

[19] V. Baryshevsky and A. Gurinovich, THz and MMW sources using "grid" photonic crystals, in Proceedings of IRMMW-THz'2007 (Joint 32nd International Conference on Infrared and Millimeter Waves and 15th International Conference on Terahertz Electronics) (Cardiff, UK, 2007), pp. 245-246, .

[20] V. G. Baryshevsky, K. G. Batrakov, and V. I. Stolyarsky, Application of volume diffraction grating for terahertz lasing in volume FEL (VFEL), Nucl. Instrum. Methods Phys. Res., Sect. A 507, 93 (2003).

[21] V. G. Baryshevsky, K. G. Batrakov, I. Ya. Dubovskaya, and S. Sytova, Visible surface quasi-Cherenkov FEL, Nucl. Instrum. Methods Phys. Res., Sect. A 358, 508 (1995).

[22] K. G. Batrakov and S. N. Sytova, Mathematical modelling of multiwave volume free electron laser, Comput. Mathematics Mathematical Phys. 45, 666 (2005).

[23] D. E. Wortman and R. P. Leavitt, Infrared and Millimeter Waves: Coherent Sources and Applications, edited by K. J. Button (Academic Press, New York, 1983), Vol. 7, Chap. 7, pp. 321-375; D. E. Wortman, C. A. Morrison, and R. P.
Leavitt, U.S. Patent No. 4545056 https://patents.justia .com/patent/4545056 (1985).

[24] R. A. Silin, Periodic Waveguides (Phasis, Moscow, 2002).

[25] R. A. Silin and V.P. Sazonov, Slow-wave structures, Moscow, Soviet Radio, 1966 (in Russian); R. A. Silin and V. P. Sazonov, Slow Wave Structures (National Lending Library, Boston Spa, 1971).

[26] L. D. Landau and E. M. Lifshitz, The Classical Theory of Fields, 4th ed. (Pergamon Press, New York, 1975).

[27] L. D. Landau, E. M. Lifshitz, and L. P. Pitaevskii, Electrodynamics of Continuous Media, 2nd ed. (Butterworth Heinemann, 1984), https://www.elsevier.com/ books/electrodynamics-of-continuous-media/landau/9780-08-057060-0.

[28] J. D. Jackson, Classical Electrodynamics, 3rd ed. (Wiley, New York, 1998).

[29] P. M. Morse and H. Feshbach, Methods of Theoretical Physics (McGraw Hill, New York, 1953).

[30] E. M. Lifshitz and L. P. Pitaevskii, Physical Kinetics: Volume 10 (Course of Theoretical Physics) (Pergamon, New York, 1981).

[31] R. J. Briggs, Electron-Stream Interaction with Plasmas (MIT Press, Cambridge, MA, 1964).

[32] V. G. Baryshevsky, I. Ya. Dubovskaya, and I. D. Feranchuk, Cherenkov instability of the bunch of charged particles moving in three-dimensional triply periodic matter, Vest. Akad. Sci. BSSR 1, 92 (1988).

[33] V. G. Baryshevsky and I. Ya. Dubovskaya, Induced X-ray radiation of an electron beam in a free electron laser with distributed feedback in a tree-dimensional spatially periodic medium, Vest. Akad. Sci. BSSR 1, 30 (1990).

[34] V. G. Baryshevsky, K. G. Batrakov, and I. Ya. Dubovskaya, Parametric (quasi-Cherenkov) x-ray FEL, J. Phys. D 24, 1250 (1991).

[35] G. S. Nusinovich, Modern Microwave and MillimeterWave Power Electronics, edited by Robert J. Barker, N. C. Luhmann, and J.H. Booske (Wiley-IEEE Press, New York, 2005).

[36] J. R. Pierce, Travelling Wave Tubes (Van Nostrand Co., Princeton, 1950).

[37] J. R. Pierce, Theory of the beam-type traveling-wave tube, Proc IRE 35, 111 (1947).

[38] J. Benford, J. A. Swegle, and E. Schamiloglu, High Power Microwaves, Series in Plasma Physics, 2nd ed. (Taylor \& Francis, London, 2007).

[39] T. C. Marshall, Free Electron Lasers (Macmillan, New York, 1985).

[40] A. Aryshev, S. Araki, M. Fukuda, K. Lekomtsev, M. Shevelev, J. Urakawa, A. Potylitsyn, and K. Sakaue, Development of advanced $\mathrm{THz}$ generation schemes at KEK LUCX facility, in Proceedings of the 10th Annual Meeting of Particle Accelerator Society of Japan, Nagoya, Japan, 2013, https://www-linac.kek.jp/mirror/www.pasj.jp/ web_publish/pasj10/proceedings/PDF/SUP0/SUP020.pdf.

[41] V. G. Baryshevsky and E. A. Gurnevich, The possibility of cherenkov radiation generation in a photonic crystal formed by parallel metallic threads, in Proceedings of the 2010 International Kharkov Symposium on Physics and Engineering of Microwaves, Milimeter and Submilimeter Waves (MSMW'10), 2010. 
[42] E. Yablonovitch, Photonic Crystals: Whats in a Name? Optics \& Photonics News (U.C. Berkeley), 2007, http:// optoelectronics.eecs.berkeley.edu/ey2007opn183.pdf.

[43] V. G. Baryshevsky, Instability of waves in a periodic medium, Dokl. Akad. Sci. BSSR 31, 1089 (1987).

[44] V. G. Baryshevsky, Surface parametric radiation of relativistic particles, Dokl. Akad. Sci. USSR 299, 6 (1988).

[45] Fifty years of x-ray diffraction: Dedicated to the international union of crystallography on the occasion of the commemoration meeting in Munich, edited by P. P. Ewald (Springer, USA, 1962), https://link.springer.com/ book/10.1007/978-1-4615-9961-6.

[46] Z. G. Pinsker, Dynamical Scattering of X-rays in Crystals (Springer-Verlag, Berlin, 1978).

[47] R. W. James, The Optical Principles of the Diffraction of $X$-rays (Ox Bow Press, 1982). 